\title{
Analyse de cartes de l'Atlas mondial suisse: survol et résumé
}

L'Atlas mondial suisse (AMS) est le seul moyen d'enseignement de géographie au niveau national en Suisse. Cet ouvrage ayant connu une nouvelle édition en 1993 (JEAN. NERET 1993), mise à jour en 1994, il s'agit d'en présenter le concept et de démontrer des applications scolaires. Le rédacteur et quelques auteurs de carte et le conseiller de la rédaction présentent dans ce numéro de Geographica Helvetica des exemples d'interprétation de cartes. Voici quelques éléments et développements, avec en particulier un choix de problèmes et de travaux d'élèves.

\section{MAX SCHÜEPP: Situations météorologiques typiques}

(AMS p. 20)

Les cartes des situations météorologiques typiques sont présentées sur le fond d'images de satellite NOAA et METEOSAT, dont les taches blanches peuvent représenter soit de la nébulosité, soit une couverture neigeuse. Il s'agit de quatre situations advectives (haut de la page), avec un apport de masses d'air de l'extérieur, et de deux situations convectives (bas de la page), avec un échange vertical dominant dans l'atmosphère.

La situation orageuse (carte à gauche en bas) est caractérisée par des gradients barométriques faibles, mais dans un contexte peu stable. L'air réchauffé monte, créant des nuages d'orage, par exemple sur le Languedoc, entraîné par une circulation en altitude jusqu'en Romandie.

Par un centre de haute pression peu accentué sur les Alpes (carte à droite en bas), les Alpes enneigées sont bien reconnaissables. Les régions basses de Bavière, la plaine du Pô et le Moyen-Pays inférieur sont recouverts de brouillard.

Les différences des températures équatoriales et polaires sont compensées par des circulations advectives. La circulation zonale, dans le cadre d'une situation de vent de l'ouest (carte du haut à droite) est fréquente durant l'hiver. La poussée d'air équatorial chaud en direction du pôle et l'écoulement d'air froid vers l'équateur sont les deux branches de la circulation méridienne (carte à gauche en haut et à gauche au milieu). La circulation septentrionale, créant au nord des Alpes un barrage avec une forte nébulosité et des précipitations, se constitue surtout durant la deuxième moitié de l'hiver. Au sud des Alpes, le ciel est serein, par fœhn du nord, tandis que le Mistral souffle sur la vallée du Rhône au sud de Lyon. Sur la carte (milieu à gauche), on reconnaît la zone de beau temps, sur la plaine du Pô.
Une méthode est proposée pour reconstituer la carte météorologique en altitude (au niveau des $500 \mathrm{hPa}$ ) et pour une interprétation des cartes en surface et en altitude.

\section{ARTHUR JETZER: Les Landes - un espace touristique français (AMS p. 35)}

La carte thématique comporte la partie centrale de cette région. Les travaux proposés permettent d'apprécier l'attraction touristique d'un espace et de reconnaître les conflits d'intérêts résultant du tourisme. La carte présente des éléments intéressants concernant le tourisme et sert à mettre en cause notre propre comportement de touriste.

1. Quelles sont les caractéristiques de cet espace?

2. Lesquelles sont importantes pour les touristes?

3. Quel est l'attrait spécifique pour le tourisme?

4. Y a-t-il sur cette carte des indices des conditions climatiques?

5. Quelles localités sont particulièrement favorables au tourisme?

6. Quels sont les types de logement touristiques offerts dans ces stations?

7. Avec quels moyens de locomotion ces destinations sont-elles atteignables?

8. Quels sont les moyens de locomotion favorables aux excursions dans ces régions touristiques?

9. Quelles sont les activités économiques les plus importantes dans cet espace?

10. $Y$ a-t-il des indications concernant l'utilisation des immenses forêts de pin?

11. Quelles sont les activités qui risquent de porter atteinte au tourisme?

\section{ROGER MEIER: Orihuela - climat, agriculture, irrigation et exportation (AMS p. 75)}

La région de Murcie figure souvent dans les atlas scolaires, mais l'Atlas mondial suisse offre une carte à grande échelle permettant de mieux présenter en détail l'habitat, l'infrastructure et le paysage physique.

François Jeanneret, Dr, Université de Berne, Institut de Géographie, Hallerstraße 12, 3012 Berne 
La répartition saisonnale des précipitations dans le bassin méditerranéen a des inconvénients: les pluies tombent surtout en hiver, mais manquent en été. La province d'Alicante, située sur la côte orientale, est particulièrement sèche. Pour cette raison, une partie du cours supérieur du Tage a été déviée dès 1979 vers la Costa Blanca. Les travaux suivants, basés sur cette carte relativement élémentaire, peuvent aussi être proposés aux élèves des écoles secondaires:

- Dessine une coupe du canal d'irrigation.

- A quelle saison les agrumes font-ils leur apparition sur le marché?

- Qu'est-ce qui est cultivé sur le reste des terres agricoles?

- Pourquoi est-il logique pour l'Espagne d'étendre la culture des agrumes?

\section{GEORG JUNG: Les bidonvilles}

Le terme de "slum» fut utilisé dans les villes industrielles au $18 \mathrm{e}$ siècle, en Angleterre, signifiant des quartiers ouvriers négligés. Equivalent de bidonvilles, ils s'appellent en espagnol «barriadas» (Pérou), "villas miserias» (Argentine) ou "callampas» (Chili), en portugais "favelas», en anglais "squatters» ou "slums», en allemand "Elendsviertel», "Primitivbauten», «Hüttenquartiere», en Inde "basti».

\section{a) Bidonvilles et quartiers rudimentaires}

Des travaux pratiques comme les suivants concernent les bidonvilles en général:

1. L'élève cherche des bidonvilles dans les légendes des cartes des villes de l'Atlas et les présente sous forme d'un tableau.

2. L'élève essaie d'estimer l'âge des bidonvilles à partir de leur situation dans le tissu urbain et de la chronologie de leur construction.

Dans l'AMS, plusieurs cartes de villes comportent des bidonvilles:

b) Lima ( $A M S$ p. 148)

1. Déterminer les proportions des surfaces des barriadas, tugurios et du reste de la surface urbaine par la méthode du quadrillage (décompte de carrés à l'aide d'un papier transparent millimétré).

2. Comment peut-on interpréter les différences de densité de l'habitat dans le centre de Lima (18 600 habitants par $\left.\mathrm{km}^{2}\right)$ et des barriadas $\left(790 \mathrm{~km}^{2}\right)$ ?

c) Coyoacán, Mexique (AMS p. 149)

1. Quels rapports y a-t-il entre les fonctions, la densité de population, le prix du sol et les revenus dans les quartiers avec constructions rudimentaires?

2. Y a-t-il une différence de desserte et d'infrastructure entre les quartiers rudimentaires et les autres?

d) Douala, Cameroun (AMS p. 91)

1. Que sont devenus les bidonvilles de 1946 au cours du développement urbain?
2. Dans quelle direction la ville s'est-elle développée en premier?

3. Pourquoi les nouveaux bidonvilles (dès 1983) furentils construits sur des terres agricoles et non pas ailleurs, par exemple au sud de Bonanloka?

4. Calculez la surface des bidonvilles et de la ville entière (selon la méthode du quadrillage décrite pour Lima).

5. Quelle a été l'évolution du rapport de surface entre les bidonvilles et la ville entière, de 1946 à 1983 ?

e) Casablanca, Maroc (AMS p. 95)

1. Situez les bidonvilles.

2. Calculez les distances pour les déplacements d'un habitant des bidonvilles travaillant dans le port ou qui est vendeur de rue (par exemple de cigarettes) dans le quartier portuaire. Quelles sont les conséquences financières du long chemin de travail?

f) Comparaison de Bombay et Jaipur (AMS p. 109)

1. Quelle est la différence entre la localisation des bidonvilles à Bombay comparé à ceux de Jaipur?

2. Pourquoi les bidonvilles de Bombay situés entre le port et les quartiers de la classe moyenne, ne sont-ils pas menacés?

3. Oủ peut-on s'attendre à un développement de nouveaux bidonvilles à Bombay?

g) Brasilia, Brésil (AMS p. 148)

1. Localiser les favelas à Brasilia.

2. Pourquoi n'y a-t-il pas de favelas sur les cartes de Gurá, Sobradinho et Tagatingua, qui pourtant - au dire des journaux - existent et se développent?

\section{PETER KOCH: Culture sur brûlis (AMS p. 91)}

Une approche générale est proposée pour l'interprétation de cartes, applicable à toutes les cartes thématiques, dont la carte de Zimgbo Town (anciennement Zumbata) au Libéria.

\section{Première approche de la carte}

1. Comprendre le thème: titre de la carte, légende. Qu'est-ce qui frappe à premier abord? Quel est le but de la carte?

2. Localisation de l'espace: délimitation, coordonnées, situation de l'espace représenté, consultation d'une carte plus générale.

3. Prendre connaissance de l'èchelle: estimer la surface du fragment de carte.

4. Lire la légende et les commentaires: qu'est-ce qui est représenté?

5. Exploration de la carte: quelle en est le sujet, quelles questions sont posées?

Description du contenu de la carte

1. Saisie du fond de carte: description de l'orographie et de l'hydrographie, la situation et le réseau des transports. Estimation du nombre d'habitants (en tenant compte de la généralisation). 
2. Comprendre le thème: de quelle manière le thème estil développé?

a) Identifier la signification des couleurs.

b) Décrire les formes des champs.

c) Quels sont les aliments produits par les agriculteurs?

d) Rechercher les rizières anciennes et leur affectation actuelle.

e) Reporter les rizières sur du papier transparent millimétré et estimer le rapport de cette surface à celle du village.

f) Quelle est la durée de l'exploitation des champs et celle de la jachère?

\section{Expliquer la carte}

(en consultant des cartes supplémentaires)

1. Saisir les causes, conclusion.

a) Dans quelle zone climatique se trouve le Libéria?

b) Chercher des diagrammes climatiques semblables à celui de la côte du Libéria.

c) Déterminer la densité de population dans les alentours de Monrovia.

d) Déterminer les sols de la région de Zimgbo Town.

e) Désigner le système agricole de Zimgbo Town.

f) Expliquer le système de cultures.

g) Comment peut-on imaginer les conditions de propriété foncière?

h) Pratique-t-on aussi de l'élevage?

2. Résumé: caractéristiques de la culture sur brûlis, cause de ce type d'utilisation du sol, avantages et inconvénients, comparaison avec d'autres cas, avec l'agriculture en Suisse. Décrire les limites de l'interprétation. Quelles sont les informations manquantes, comment pourrait-on les trouver?

La culture sur brûlis itinérante («Shifting Cultivation») est une forme d'utilisation du sol ancienne, très répandue dans les zones intertropicales à faible productivité.

\section{PETER KOCH: Pays en voie de développement sahéliens} (AMS p. 90, 92-94, 96, 97)

Trois types d'enseignement sont envisageables avec un atlas:

1. Centré sur l'orientation

a) Connaissances topographiques

b) Présentation de systèmes spatiaux (vue globale, par exemple structure zonale, politique, culturelle ou économique)

2. Centré sur l'espace: géographie régionale, étude de cas

3. Centré sur des thèmes: travail exemplaire et thèmatique

Voici un choix de problèmes proposés, par différentes catégories:

\section{Problèmes d'orientation}

- Qu'est-ce le Sahel et où se trouve-t-il?

- Esquissez la zone du Sahel. Quelle sont ses dimensions?
- Quels états se départagent cet espace?

- Cite les capitales de ces états.

- Quels cours d'eau peut-on évoquer?

Elaboration de la géographie physique

- Décrire le relief et les altitudes du Sahel.

- Quel vent souffle durant la période sèche, comment s'appelle-t-il en Afrique occidentale?

- Dans quelle zone climatique se trouve Niamey?

- Quels sont les sols les plus fréquents du Sahel?

- Quels sont les dangers naturels qui touchent le Sahel?

Population, habitat

- Quelle est la répartition de la densité de population?

- Quelle est la dynamique de la population?

- Quelle est la structure de l'emploi, et quel est le pourcentage des agriculteurs?

Structure ethnique et histoire

- Quelle est la religion dominante?

- Dessine sur du papier calque les voies de communication du ler siècle av. J.-C. et des Arabes.

- D'où provenait l'or qui était commercialisé dans le passé et qui est à nouveau exploité?

- Quand et par quel pays le Sahel occidental fut-il colonialisé?

Utilisation du sol et nutrition

- Quel type d'agriculture est dominant?

- De quelles céréales les Sahéliens se nourissent-ils?

- Quels produits d'exportation peut-on cultiver?

- Quelle est la répartition des dattiers? Y en a-t-il encore actuellement?

- Quels sont les animaux des nomades et des seminomades au sud et au nord du Sahel?

Réseau des transports

- Analyse les transports dans le Sahel.

- Quel est le degré de motorisation?

Structure économique et sociale

- Quelle est la productivité de l'économie en comparaison mondiale?

- Quel est le développement de l'industrie dans les états du Sahel?

- Combien de médecins y a-t-il par exemple au Mali et au Tchad?

- De quelles maladies les habitants souffrent-ils?

\section{ERNST SPIESS: Les cartes économiques}

Lors de l'élaboration d'un concept pour les cartes économiques, préférence fut donnée aux cartes complexes réunissant un nombre important d'éléments. En les jumelant avec des cartes générales à même échelle, on a voulu faciliter la lecture et l'interprétation.

Les cartes économiques comportent les éléments de la végetation de manière sommaire, par contre l'utilisation du sol est différentiée. Les produits de l'agriculture et de la sylviculture sont représentés par un nombre de sym- 
boles, auxquels s'ajoutent ceux pour les mines, l'industrie et les services, en couleurs vives. Les symboles des villes, des mines et de la production industrielle sont échelonnés en plusieurs grandeurs, afin de démontrer leur importance relative.

La quantification se fait, pour toutes les cartes à échelle identique, de la même manière, pour faciliter les comparaisons. Ainsi, la carte économique de l'Europe centrale (pages 50/51 de l'Atlas) peut paraître un peu surchargée, mais elle reflète fidèlement les conditions réelles. L'estompage peut parfois gêner un peu l'identification des couleurs, mais est d'un intérêt incontestable pour l'interprétation des cartes thématiques.

\section{Bibliographie}

JEANNERET, F. (1993): L'Atlas mondial suisse: une ancienne tradition et une nouvelle édition. Dans: Bulletin de la Société neuchâteloise de géographie 37, p. 7-16.

JETZER, A., JUNG, G., KOCH, P., MEIER, R., SCHÜEPP, M., SPIESS, E. (1996): dans Geographica Helvetica 1/96.

\section{Literaturbesprechungen}

DANSON Mark F./ PLUMMER Stephen E. (Edits.): Advances in Environmental Remote Sensing. 184 S., zahlr. Fig. und Tab., Verlag John Wiley \& Sons Ltd., Chichester, 1995, $£$ 55.--.

Dieser Sammelband mit 11 Beiträgen renommierter Fernerkundler entspringt einer Tagung der "British Geographers", an der versucht wurde, die Zusammenhänge zwischen Objekten und Prozessen an der Erdoberfläche mit den technischen Fortschritten der Fernerkundung besser verstehen und meistern zu lernen. Insbesondere sollten die aktuellsten Forschungs- und Entwicklungstendenzen zur Lösung von Umweltproblemen vorgestellt und diskutiert werden. Die Fortschritte im Einsatz der Fernerkundung in Kombination mit anderen methodischen Verfahren ganz allgemein und speziell für umweltrelevante Anwendungen von der regionalen bis zur globalen Ebene sind enorm und nur noch schwierig zu überblicken. Eine derartige Tagung samt entsprechender schriftlicher Dokumentation ist deshalb sehr begrüssenswert und wichtig. Entscheidend ist natürlich, dass es gelingt, die bestqualifizierten Experten zusammenzubringen und eine ausgewogene qualitativ hochwertige Auswahl treffen zu können. Dies ist recht gut gelungen, auch wenn naturgemäss britische Autoren stark dominieren. Das Niveau der einzelnen Beiträge darf, wenn sicher nicht als konsistent, so doch als allgemein recht hoch und die Fernerkundung breit abdeckend eingestuft werden.

Einerseits kommen die neuesten Technologien wie Bild- und Feldspektrometrie, SAR-Polarimetrie und Interferometrie, der Informations-Extraktion etc. als auch die parallel dazu erfolgten Neuerungen und Trends in den thematischen, praxisorientierten Applikationen in gut verständlicher Form zur Sprache. Rund die Hälfte der Beiträge befasst sich mit den technischen, theoretischen und methodischen Grundlagen. Die andere Hälfte geht von den thematischen Aspekten wie biophysikalische Parameter der Pflanzendecke, Biomasse und landwirtschaftliche Produktion, geomorphologische Prozesse, Wasserqualität etc. aus.

Sollte ein einzelner Artikel besonders hervorgehoben werden, so würde meine Wahl auf denjenigen von QUEGAN über "Recent Advances in Understanding SAR Imagery" fallen. Müssten die gravierendsten Lücken identifiziert werden, so kommen auf der technischen Seite die passiven Mikrowellen und ihre immensen Möglichkeiten bei der globalen Meeres- und Atmosphärenerforschung, sowie auf der thematischen Seite Aspekte des Monitorings von Atmosphärenverschmutzung, Niederschlag und Verdunstung zuwenig zur Sprache.

Gesamthaft gesehen liegt ein besonders für umweltengagierte Wissenschaftler und Praktiker, die bereits über gute Kenntnisse in der Fernerkundung verfügen, inhaltsreiches und anregendes Buch vor, das hinsichtlich aktuellem Stand der Forschung und Zukunftsperspektiven für die Umsetzung in die praktische Anwendung eine klare Standortbestimmung vornimmt.

\section{GH 1/96} Harold Haefner, Zürich 